\title{
MO-THEORETISCH KONZIPIERTE KANDIDATEN FÜR EIN NEUARTIGES KONZEPT EINER LICHTINDUZIERTEN ELEKTRONENPUMPE
}

Wolf-Dieter Stohrer* und Rainer Paape

Studiengang Chemie der Universilät, D-2800 Bremen

Franz Effenberger und Peter Bäuerle

Institut für Organische Chemie, Biochemie und Isotopenforschung

der Universität, D-7000 Stuttgart

Summary: MO-theory suggests $\underline{3}$ as a likely candidate for a newly designed conception to replace a given redox potential by a lower one with the aid of light.

Die bei kleinen Brücken $X$ aus sterischen Gründen nur disrotatorisch mögliche elektrocyclische Reaktion $\underline{1} \underline{2}$ erfüllt auf Anhieb vier der fünf Bedingungen, die an eine pericyclische Reaktion gestellt werden müssen, damit sie als möglicher Kandidat für ein neuartiges Konzept einer lichtunterstützten Elektronenpumpe in Frage kommt /1/:

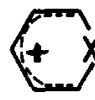

1

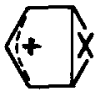

$\underline{\mathbf{2}}$

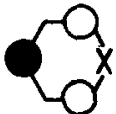

LUMO1

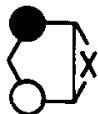

LUMO 2

- die Lage des thermischen Gleichgewichtes liegt auf der Seite von $\underline{1}$ bzw. $\underline{1}^{-}$;

- die disrotatorische Ringschlußreaktion $\underline{1} \rightarrow \underline{2}$ ist photochemisch symmetrieerlaubt;

- die disrotatorische Ringöffnungsreaktion $\underline{2} \rightarrow \underline{1}$ ist thermisch symmetrieverboten;

- das thermische Symmetrieverbot der Ringöffnung $\underline{2} \rightarrow \underline{1}$ wird durch Reduktion von $\underline{2}$ zum bicyclischen Radikal deutlich gelockert.

Die Bedingung aber, daß das LUMO von 1 energetisch über dem von $\underline{2}$ liegen muß $/ 1 /$, ist nicht erfüllt: Das LUMO von $\underline{1}$ und das von $\underline{2}$ sind nichtbindende und damit praktisch energiegleiche Orbitale. Durch ein geeignetes Substitutionsmuster läßt sich aber auch diese Bedingung erfüllen:

- ein Donor D an der Position 1, 3 und/oder 5 hebt das LUMO von 1 (mit hoher Koeffizientendichte in diesen Positionen) energetisch an, ohne das LUMO von $\underline{2}$ (ohne Koeffizientendichte in diesen Positionen) zu beeinflussen;

- ein Akzeptor A an der Position 2 und/ader 4 senkt das LUMO von 2 (mit hoher Koeffizienten- 
dichte in diesen Positionen) energetisch ab, ohne das LUMO von $\underline{1}$ (ohne Koeffizientendichte in diesen Pusitiunen) zu beeinrlussen.

Durch unterschiedliche Art und Anzahl dieser Substituenten läßt sich dic cnergctische Lage des (höherliegenden) LUMO des Monocyclus und des (tieferliegenden) LUMO des Bicyclus sowie die Energie der längstwelligen Lichtabsorbtion über einen weiten Bereich variieren /2/. Das Mndellsystem $3 \approx 2-4$ verdeutliche das Konzept einer neuartigen Iichtunterstützten Elektronenpumpe:

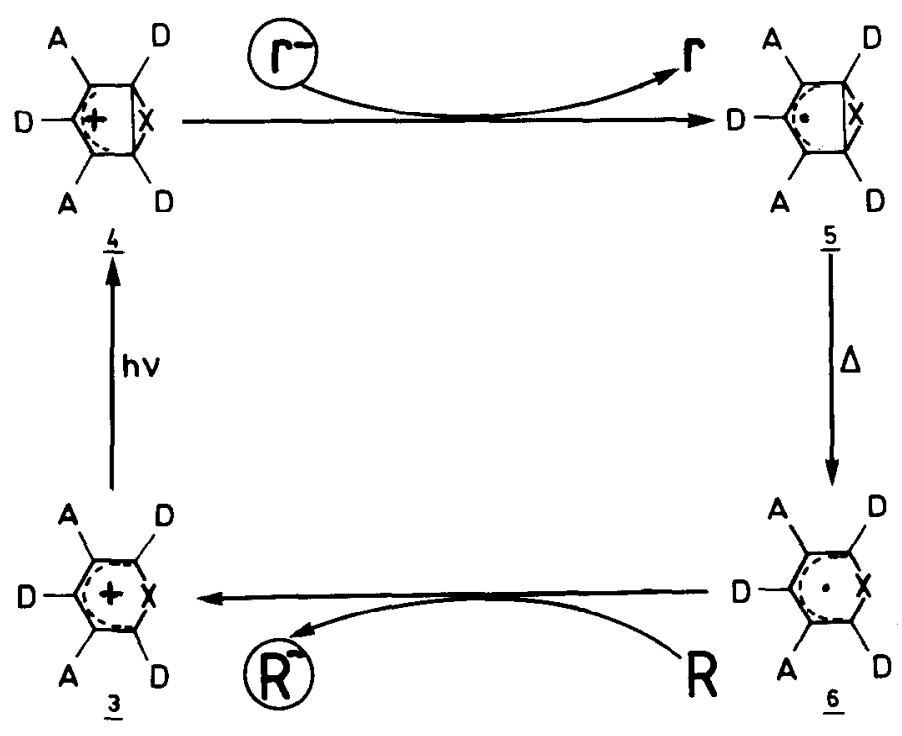

Der Monocyclus $\underline{3}$ (mit energetisch hochliegendem LUMO) schließt lichtinduziert (symmetrieerlaubt) zum Bicyclus 4, der (aufgrund seines energetisch tiefliegenden LUMO) bereits durch ein schwaches Reduktionsmittel $\mathrm{r}^{-}$, das zur Reduktion von $\underline{3}$ nicht ausreicht, zum bicyclischen Radikal $\underline{5}$ reduziert werden kann, das thermisch ("halberlaubt") zum monocyclischen Radikal 6 mit energetisch hochliegendem SOMO (und folglich mit im Vergleich zu $\underline{5}$ erniedrigtem Redoxpotential) öffnet /3/. Das Radikal 6 kann das vom schwachen Reduktionsmittel $\Gamma^{-}$nicht reduzierbare $R$ zum starken Reduktionsmittel $\mathrm{R}^{-}$reduzieren unter Rückbildung des monocyclischen Kations $\underline{3}$, das erneut in den Kreislauf eintreten kann /4/. Das schwache Reduktionsmittel $\mathrm{r}^{-}$wird also lichtunterstützt durch das starke Reduktionsmittel $\mathrm{R}^{-}$ersetzt.

/1/ Wolf-Dieter Stohrer, vorstehend

/2/ MO-Rechnungen, W.-D. Stohrer und R. Paape, unveröffentlicht

13/ 1t. MND0-Rechnungen liegen die Gleichgewichte $\underline{3} \approx \underline{4}$ und $\underline{6} \not \underline{5} \underline{5}$ für $X=\mathrm{CH}_{2}, \mathrm{D}=\mathrm{NH}_{2}, \mathrm{~A}=\mathrm{H}$ und für $X=\left(\mathrm{CH}_{2}\right)_{2}, \mathrm{D}=\mathrm{H}, \mathrm{A}=\mathrm{CN}$ jewseils auf der linken Seite

/4/ Eine mit der Oxidation $\underline{6} \stackrel{-\mathrm{E}}{\longrightarrow} \underline{3}$ möglicherweise konkurrierende Dimerisation von $\underline{6}$ zu $\underline{6}_{2}$ sollte nicht schädlich sein, da derartige Dimerisierungen üblicherweise reversibel sind, so daß sich das Gleichgewicht $\underline{6}+\underline{6}(6) 2$ - dessen Lage durch große Substituenten $D$ bzw. A nach links verschoben werden kann - bei der Oxidation von $\underline{6}$ zu $\underline{3}$ sofort wieder einstellt.

(Received in Germany 24 December 1984) 